\title{
The optimal solution for unit commitment problem using binary hybrid grey wolf optimizer
}

\author{
Ali Iqbal Abbas, Afaneen Anwer \\ Department of Electrical Engineering, University of Technology, Baghdad, Iraq
}

\begin{tabular}{l}
\hline \hline Article Info \\
\hline Article history: \\
Received Mar 22, 2021 \\
Revised Jun 15, 2021 \\
Accepted Jul 2, 2021 \\
\hline
\end{tabular}

Keywords:

Economic dispatch Generation scheduling Grey wolf optimizer Particle swarm optimization Unit commitment

\section{Corresponding Author:}

Ali Iqbal Abbas

Department of Electrical Engineering, University of Technology

Baghdad, Iraq

Email: eee.19.14@grad.uotechnology.edu.iq

\begin{abstract}
The aim of this work is to solve the unit commitment (UC) problem in power systems by calculating minimum production cost for the power generation and finding the best distribution of the generation among the units (units scheduling) using binary grey wolf optimizer based on particle swarm optimization (BGWOPSO) algorithm. The minimum production cost calculating is based on using the quadratic programming method and represents the global solution that must be arriving by the BGWOPSO algorithm then appearing units status (on or off). The suggested method was applied on "39 bus IEEE test systems", the simulation results show the effectiveness of the suggested method over other algorithms in terms of minimizing of production cost and suggesting excellent scheduling of units.
\end{abstract}

This is an open access article under the CC BY-SA license.

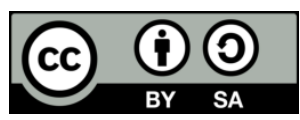

\section{INTRODUCTION}

Unit commitment is a significant issue in both the operation of electrical power systems and the competitive electricity supply industry and market. It includes two decision-making processes: unit scheduling and economic dispatch (ED). The generators scheduling process involves setting the status of generating units every hour to be (ON or OFF) of the horizon planning while taking into account the constraints of the units' minimum up and down-time. Process of determining the best power generation of generation plants (units) to meet power demand and spinning reserve at every hour at the lowest production cost within capacity unit limits using economic dispatch goals [1].

Because of load variation problem through the day, and for large power systems which contain many plants or units, it is not suitable to operate all plants in time. So a method of coordination among these plants and generating units must be adopted. The coordination involves in which units will be a start-up and the sequence of operating units that must be connected to the network (i.e. ON) and which must be switched OFF. Another problem must be solved that it produces electrical power with fulfilling demands in a minimum cost [2].

Many methods have been used to solve the unit commitment (UC) problem as integer programming [3], branch-and-bound methods [4], dynamic programming (DP) [5]-[8], mixed-integer programming [9], and lagrangian relaxation methods (LR) [10], [11], and priority list method [12]. Also, there are many optimization algorithms suggested to solve unit commitment as whale optimization-differential evolution and genetic algorithm (WODEGA) [13], on mixed-integer programming formulations for the unit commitment problem [14], solving unit commitment and economic load dispatch problems using modern optimization algorithms [15], sested particle swarm optimization (PSO) [16], dynamic formulation of the unit commitment 
and economic dispatch problems [17]. In this paper the optimal UC solution based on binary grey wolf optimizer based on particle swarm optimization (BGWOPSO) for (39 bus IEEE system with 10 units) has been presented in discussion and the results compared with related works.

\section{RESEARCH METHOD}

Unit commitment (UC) issues are often used to assess which units must participate in dispatch operations at particular times. The number of units, load capacity, startup cost, spinning reserve, and ramp rate are all constraints that must be considered. The goal is to create a secure UC system that meets the aforementioned constraints at a low total cost. According to their characteristics, power generating systems may be listed as base-load, intermediate-load, or peak-load, so the main constraints can be listed as follows:

\subsection{Constraints}

In order to solve UC problem, the constraints must be satisfied which are imposed as operational requirements. The constraints can be explained as following [1], [18]:

a. Power constraint

The sum of power generation of each generator at time $\mathrm{t}\left(P_{g i}^{t}\right)$ equal to demanded power:

$$
\sum_{i=1}^{N g} P_{g i}^{t}=P_{D}^{t}
$$

where $P_{D}^{t}$ represents active power which demanded at time $\mathrm{t}$ and $\mathrm{Ng}$ number of generators.

b. Spinning reserve constraint

This term is used to characterize the total maximum active generated power from all units in the system minus the current load demand. In other words, mathematically we can say:

$$
\sum_{i=1}^{N g} P_{g i(\operatorname{Max})} \geq P_{D}^{t}+P_{R}^{t}
$$

where $P_{g i(\max )}$ represents the maximum real power of the unit (i), and $P_{R}^{t}$ is the reserve of real power at time t. c. Power limit constraints

$P_{g i(\min ) \text { is }}$ is the minimum active power of unit $i$.

$$
P_{g i(\min )} \leq P_{g i}^{t} \leq P_{g i(\max )}
$$

d. Minimum up time constraint

$T_{i, \text { on }}^{t}$ is the number of hours that unit (i) is on till time t, and MUTi is the minimum uptime of unit (i).

$$
T_{i, o n}^{t} \geq M U T_{i}
$$

e. Minimum down time constraint

$T_{i, o f f}^{t}$ represents off hours of unit (i) till time t, and MDTi is the minimum downtime of unit (i).

$$
T_{i, o f f}^{t} \geq M D T_{i}
$$

In unit commitment, the priority list to make what unit is $\mathrm{ON}$ is based on the rate of the cost of fullload $(\gamma)$ for a unit which can define as the fuel cost divided on generated power at the maximum value of the unit [1].

$$
\begin{aligned}
& \gamma_{i}=\frac{f_{i}}{P_{\text {gimax }}} \\
& \gamma_{i}=\mathrm{a}_{i} P_{\text {gimax }}+b_{i}+\frac{c_{i}}{P_{\text {gimax }}}
\end{aligned}
$$

Where a unit that has $\gamma_{i}$ the least with respect to other units will be prioritized to dispatch its power firstly. The scheduling of units perhaps does not satisfy the constraint of spinning reserve. Therefore must solve this violation that occurred. One approach that found to this purpose is depending on $\gamma_{i}$ as following [1]:

a) At each time hour, find $\gamma_{i}$ via (7) for each uncommitted unit at hour t, then sort them in an ascending form.

b) Find the requirement of the spinning reserve at $t$ by (2). 
c) In case the result occurred in point (2) achieves the constraint of spinning reserve, check $\mathrm{t}$, if $\mathrm{t}<\mathrm{T}$, go to point 1; otherwise, end this process. In the event point 2 is not achieved, one of the uncommitted units must be committed that has the least $\gamma_{i}$ from point (1).

\subsection{The economic dispatch}

The main constraint of the operating system is the summation of the active output power of units equal to power demand. The input to every unit, shown as $f i$, where $f i$ refers to the cost rate of operation of the unit (i) which is the fuel cost. The output of every unit, Pgi, is the active power generated by the ith unit. The total cost represents the summation of the cost of each individual unit which mathematically denoted $F_{T}$. So the problem of economic dispatch is minimizing $F_{T}$. The fuel cost $f_{i}$ of unit (i) at any interval time is a function for the power generated of generating unit output $P g_{i}$ [19].

$$
\left(f_{i}^{t}=c_{i}+b_{i} P_{g i}+a_{i} P_{g i}^{2}\right)
$$

Where $\mathrm{a}_{\mathrm{i}}, \mathrm{b}_{\mathrm{i}}$, and $\mathrm{c}_{\mathrm{i}}$ are the fuel cost coefficients. The unit has incremental fuel cost $(\lambda i)$ which considered the slope of the input-output curve and equal $\frac{\partial f i}{\partial P_{g i}}=\lambda i$. So, for the economical partition of load among units within the plant, the norm is that all units must be operating at the same incremental fuel cost.

\subsection{Hybrid grey wolf optimizer base on particle swarm optimization \\ 2.3.1. Particle swarm optimization}

Particle swarm optimization (PSO) is the algorithm presented by Kennedy and Eberhart [20]. This theory was developed relying mainly on the intelligence of the swarm to do this advanced computational procedure. In a swarm, every particle is considered a feasible solution to the optimization problem. The movement of particles in space depends on the rules of flow. In the case of one of the particles discovering a solution, the other particles will approach it. For all individuals, the values of fitness are estimated and that will be optimized through the function of fitness. As a PSO is a tool of optimization, it provides an exploration process in which residents constantly change their locations over time. Through investigation, each particle maintains the best position it found, $\mathrm{P}_{\text {best. }}$ In addition, among all the $\mathrm{P}_{\text {best }}$ values gained the better fitness is specific by one particle, which is known as the global best, known $\mathrm{G}_{\text {best }}$ [21].

The position of the particle and velocity must be updated. Based on the fitness values the personal location $\mathrm{P}_{\text {best }}$ and global location $\mathrm{G}_{\text {best }}$ are reconstituted as in (9)-(11) [1]:

$$
\begin{aligned}
& V_{i}^{t+1}=w^{t} V_{i}^{t}+c_{1} r_{1}^{t}\left(P_{\text {best }}^{t}-x_{i}^{t}\right)+c_{2} r_{2}^{t}\left(G_{\text {best }}^{t}-x_{i}^{t}\right) \\
& x_{i}^{t+1}=x_{i}^{t}+V_{i}^{t+1} \\
& w^{t}: \text { Inertia weight factor }=w_{\text {max }}-\frac{w_{\text {max }}-w_{\min }}{\text { Iter }_{\max }} \times \text { Iter }
\end{aligned}
$$

where: "c $c_{1}, c_{2}$ : acceleration coefficient", " $\mathrm{x}$ : the location of particle", " $\mathrm{r}_{1}, \mathrm{r}_{2}$ : Two numbers taken randomly

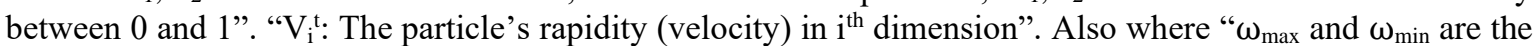
initial and final weights and iter $_{\max }$ is the maximum iteration number".

\subsubsection{Grey wolves optimizer (GWO)}

This method relies on grey wolves' behavior in obtaining their food, as grey wolves are at the topmost of predators [22]. The hierarchy of grey wolves is the leader in the herd is called alpha $(\alpha)$ and is responsible for giving decisions. The second level is beta $(\beta)$ wolves. The third and lower ranks of the pack are delta $(\delta)$ and omega $(w)$ respectively. Alpha is the fittest solution, both beta and delta will be the second and third best solutions respectively [22]. Hunting prey begins first with the process of surrounding prey (encircling), which can be modeled mathematically as in (12) and (13) [22]:

$$
\begin{aligned}
& \vec{D}=|\vec{C} \cdot \vec{X} p(k)-\vec{X} w(k)| \\
& \vec{X} w(k+1)=\vec{X} p(k)-\vec{A} \cdot \vec{D}
\end{aligned}
$$

where "k: the present iteration", " $\vec{A}$ and $\vec{D}$ : Coefficient vectors", “ $\vec{X} p$ : The position vector of prey", " $\vec{X} w$ : The position vector of the grey wolf". It can calculate the vectors $\vec{A}$ and $\vec{C}$ as in (14) and (15): 


$$
\begin{aligned}
& \vec{A}=2 \vec{a} \cdot \overrightarrow{r_{1}}-\vec{a} \\
& \vec{C}=2 \cdot \overrightarrow{r_{2}}
\end{aligned}
$$

where throughout iterations components " $\vec{a}$ are decreased from 2 to 0 linearly" and " $\left(\overrightarrow{r_{1}}, \overrightarrow{r_{2}}\right)$ are random vectors between $[0,1]$ ”.

Gray wolves can modify their position about the prey [23] and have the capability to distinguish the prey's location. The hunting process is usually alpha-guided. To mathematically represent hunting behavior, it suggests that alpha, beta, and delta have the best known probable site of the prey [22]. Therefore, the first three optimal solutions it was obtained are kept to update their sites in the space proportional with the optimal place [24], and the other solutions, including Omega solutions, are neglected [22]. These solutions can be determined as in (16) [22].

$$
\overrightarrow{D_{\alpha}}=\left|\overrightarrow{C_{1}} \cdot \overrightarrow{X_{\alpha}}(k)-\vec{X}\right|, \quad \overrightarrow{D_{\beta}}=\left|\overrightarrow{C_{2}} \cdot \overrightarrow{X_{\beta}}(k)-\vec{X}\right|, \quad \overrightarrow{D_{\delta}}=\left|\overrightarrow{C_{3}} \cdot \overrightarrow{X_{\delta}}(k)-\vec{X}\right|
$$

And by the following formulation can calculate the prey position vector with regard to $(\alpha, \beta$, and $\delta$ ) respectively [22], [23]:

$$
\overrightarrow{x_{1}}=\overrightarrow{X_{\alpha}}-\overrightarrow{A_{1}} \cdot\left(\overrightarrow{D_{\alpha}}\right), \overrightarrow{x_{2}}=\overrightarrow{X_{\beta}}-\overrightarrow{A_{2}} \cdot\left(\overrightarrow{D_{\beta}}\right), \overrightarrow{x_{3}}=\overrightarrow{X_{\delta}}-\overrightarrow{A_{3}} \cdot\left(\overrightarrow{D_{\delta}}\right)
$$

where " $\overrightarrow{x_{1}}, \overrightarrow{x_{2}}, \overrightarrow{x_{3}}$ are a three best solutions (wolves) in the pack at the given iteration $\mathrm{k}$ ". By taking the average of $\overrightarrow{x_{1}}, \overrightarrow{x_{2}}, \overrightarrow{x_{3}}$, it can calculate the best position as the formulation (18) [23].

$$
\vec{X}(k+1)=\frac{\overrightarrow{x_{1}}+\overrightarrow{x_{2}}+\overrightarrow{x_{3}}}{3}
$$

As mentioned earlier $\vec{a}$ are decreased from 2 to 0 linearly proportional with the number of iterations, the algorithm has been updated by the (19) to calculate $\vec{a}[24]$ :

$$
\vec{a}=2-k \cdot \frac{2}{\max i t e r}
$$

where $\mathrm{k}$ : an iteration number, max iter: number of total iterations.

\subsubsection{The hybridization}

Thus, to hybridize the two meta-heuristic methods: GWO and PSO, the exploitation capability in PSO and the exploration capability in GWO will be improved to get the strength of the two variables together [25]. The first three optimal solutions are modified by the control the exploitation and exploration of the grey wolves in the search area by inertia weight factor as in (20) [25]:

$$
\overrightarrow{D_{\alpha}}=\left|\overrightarrow{C_{1}} \cdot \overrightarrow{X_{\alpha}}(k)-w \cdot \vec{X}\right|, \quad \overrightarrow{D_{\beta}}=\left|\overrightarrow{C_{2}} \cdot \overrightarrow{X_{\beta}}(k)-w \cdot \vec{X}\right|, \quad \overrightarrow{D_{\delta}}=\left|\overrightarrow{C_{3}} \cdot \overrightarrow{X_{\delta}}(k)-w \cdot \vec{X}\right|
$$

Then velocity and position equations in (9) and (10) respectively in hybrid GWOPSO will be [25]:

$$
\begin{aligned}
& V_{i}^{t+1}=w^{t}\left(V_{i}^{t}+c_{1} r_{1}^{t}\left(x_{1}-x_{i}^{t}\right)+c_{2} r_{2}^{t}\left(x_{2}-x_{i}^{t}\right)+c_{3} r_{3}^{t}\left(x_{3}-x_{i}^{t}\right)\right. \\
& x_{i}^{t+1}=x_{i}^{t}+V_{i}^{t+1}
\end{aligned}
$$

\subsection{Proposed method hybrid binary GWO based on PSO}

Because of the algorithm hybrid GWOPSO is unable to solve the problem of feature selection since it is binary problem by its nature, consequently, a binary version of the hybrid PSOGWO must be grown to be appropriate for the feature selection problem [24]. Formulation (20) shown the modifying mechanism of wolves be a function of three vectors' sites that is $x_{1}, x_{2} ; x_{3}$ and it promotes each wolf to the first three best solutions, the updating of position in (18) can be upgraded into (23) [24]:

$$
x_{d}^{k+1}=\left\{\begin{array}{l}
1 \text { if sigmoid }\left(\frac{\overrightarrow{x_{1}}+\overrightarrow{x_{2}}+\overrightarrow{x_{3}}}{3}\right) \geq \text { rand } \\
0 \quad \text { otherwise }
\end{array}\right.
$$


where " $x_{d}^{k+1}$ : the binary position which updated at kth iteration in dimension $d$ " "rand: a random number pulled from the uniform distribution $\in[1,0]$ " and sigmoid (a) is denoted as in (24) [24]:

$$
\operatorname{sigmoid}(a)=\frac{1}{1+e^{-10(x-0.5)}}
$$

$x_{1}, x_{2}, x_{3}$ in (17) are updated and they are calculated by using formulation (25) [24]:

$$
\begin{aligned}
& x_{1}^{d}=\left\{\begin{array}{lr}
1 & \text { if }\left(x_{\alpha}^{d}+\text { bstep }_{\alpha}^{d}\right) \geq 1 \\
0 & \text { otherwise }
\end{array}\right. \\
& x_{2}^{d}= \begin{cases}1 & \text { if }\left(x_{\beta}^{d}+\text { bstep }_{\beta}^{d}\right) \geq 1 \\
0 & \text { otherwise }\end{cases} \\
& x_{3}^{d}= \begin{cases}1 & \text { if }\left(x_{\delta}^{d}+\text { bstep } \text { b }_{\delta}^{d}\right) \geq 1 \\
0 & \text { otherwise }\end{cases}
\end{aligned}
$$

where $x_{\alpha, \beta, \delta}^{d}$ the position's vector of (alpha, beta, and delta) wolves in dimension $d$.

bstep $p_{\alpha}^{d}$ : a binary step in dimension $d$ that can be denoted as in (26) [24]:

$$
\text { bstep }{ }_{\alpha, \beta, \delta}^{d}=\left\{\begin{array}{lr}
1 & \text { if } \operatorname{cstep}_{\alpha, \beta, \delta}^{d} \geq \text { rand } \\
0 & \text { otherwise }
\end{array}\right.
$$

where rand: a random number pulled from the uniform distribution $\in[1,0],(d)$ : refers to dimension, $\operatorname{cstep}_{\alpha, \beta, \delta}^{d}$ : Value of the d's continuous and can be calculated by the next equation [24]:

$$
\operatorname{cstep}_{\alpha, \beta, \delta}^{d}=\frac{1}{1+e^{-10\left(A_{1}^{d} D_{\alpha, \beta, \delta}^{d}-0.5\right)}}
$$

Thus after update the best three solution positions through (25), in BGWOPSO the exploitation and exploration are controlled by an inertia weight factor as in (28) [23]:

$$
\overrightarrow{D_{\alpha}}=\left|\overrightarrow{C_{1}} \cdot \overrightarrow{X_{\alpha}}(k)-w \cdot \vec{X}\right|, \quad \overrightarrow{D_{\beta}}=\left|\overrightarrow{C_{2}} \cdot \overrightarrow{X_{\beta}}(k)-w \cdot \vec{X}\right|, \quad \overrightarrow{D_{\delta}}=\left|\overrightarrow{C_{3}} \cdot \overrightarrow{X_{\delta}}(k)-w \cdot \vec{X}\right|
$$

Accordingly, the velocity and positions have been updated as in (29) [24]:

$$
V_{i}^{t+1}=w^{t}\left(V_{i}^{t}+c_{1} r_{1}^{t}\left(x_{1}-x_{i}^{t}\right)+c_{2} r_{2}^{t}\left(x_{2}-x_{i}^{t}\right)+c_{3} r_{3}^{t}\left(x_{3}-x_{i}^{t}\right)\right.
$$

Note that in (29) the best three solutions $x 1, x 2, x 3$ are updated according to (25) [24]:

$$
x_{i}^{t+1}=x_{d}^{k+1}+V_{i}^{t+1}
$$

where $x_{d}^{k+1}$ and $V_{i}^{t+1}$ are calculated based on (23) and (29) respectively [24].

\subsubsection{Unit commitment program structure using BGWOPSO}

a. Initially, configured particles randomly and input other parameters as iteration number, c1, c2, and w. within acceptable limits of generated power ( $\mathrm{p}_{\min }$ and $\mathrm{p}_{\max }$ ).

b. Calculate production cost $\left(f_{i}\right)$ and all ED requirements as shown in section (2.3).

c. Compare the present $\left(f_{i}\right)$ for each particle with $\mathrm{P}_{\text {best. }}$ If the present $\left(f_{i}\right)$ is superior to $\mathrm{P}_{\text {best }}$, then this value is $P_{\text {best }}$ otherwise $P_{\text {best }}$ same. Then determine $G_{\text {best }}$ among $P_{\text {best }}$

d. Take the best site in (23) as search agent (individual) current site and modify the site of $\alpha, \beta, \delta$ wolves

e. Take the site of alpha as the final site of the swarm and the alpha result as the best fitness.

f. Update the speed of each individual from (29).

g. Revise the position of individual $x_{i}^{t}$ using (30).

h. If the iterations number arrives at the maximum number go to stride (9), else back to stride (3).

i. Determine the final cost of all combinations, division of power among the units, and trace the units' scheduling.

Engender the best value of $\mathrm{G}_{\text {best }}$ means that it is the best power that can be generated from each unit with the lowest total generation cost. Figure 1 presents the above steps through flow chart. 


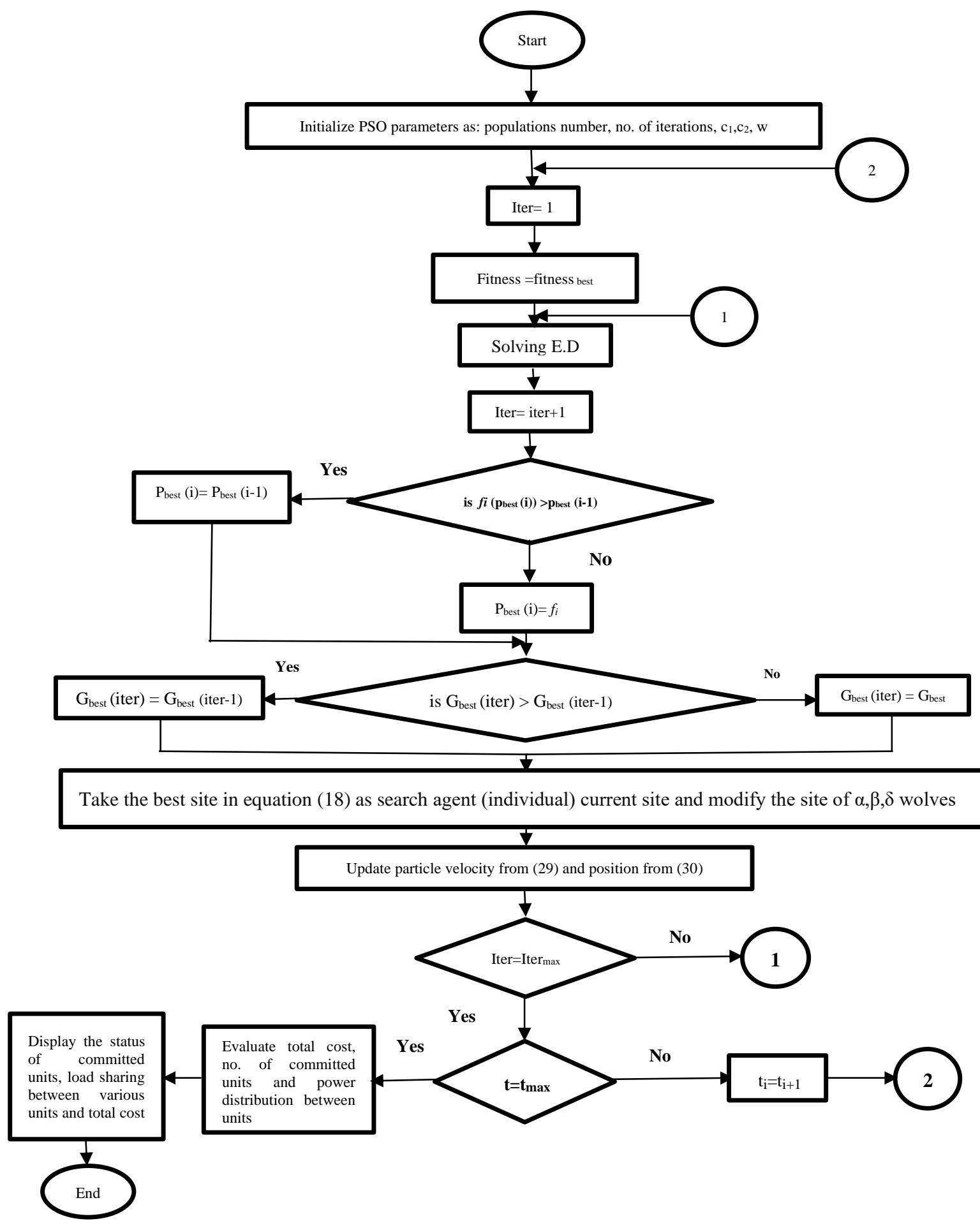

Figure 1. Flow chart of BGWOPSO algorithm in order to solve UC problem

\section{RESULTS AND DISCUSSION}

The proposed algorithm in this paper was applied on standard test system which is: 39 bus IEEE, 10 generating units shown in Figure 2. The date of the test system is presented in Tables 1 and 2 presents the daily load for the test system also. The program for this algorithm was built using MATLAB tools environment. The optimal parameters of PSO which used to obtain the best result are $\mathrm{c}_{1}=1, \mathrm{c}_{2}=1, \mathrm{w}_{\text {mini }}=0.4$, $\mathrm{W}_{\text {maxi }}=0.9$, and the number of populations is 50 with spinning reserve is 0.1 from load demand.

The results that arrived of simulating the suggested algorithm based on the test system (IEEE 39-bus) will be shown in Table 3 (see in appendix) where the units that participate in the operating system are recognized in ping color. The final production cost for this case during 24 hours is $(563940$ \$). The 
results of the IEEE 39-bus test system are compared with related works that using the same standard test system under the same conditions (i.e 0.1 spinning reserve and same constraints) as shown in Table 4 (see in appendix). It is very clear that the simulation results show the activity of the suggested algorithm BGWOPS in the reduction of the operating cost by $(0.046939 \%-0.003294 \%)$ respectively, which proves the superiority of BGWOPSO over the other optimization approaches.

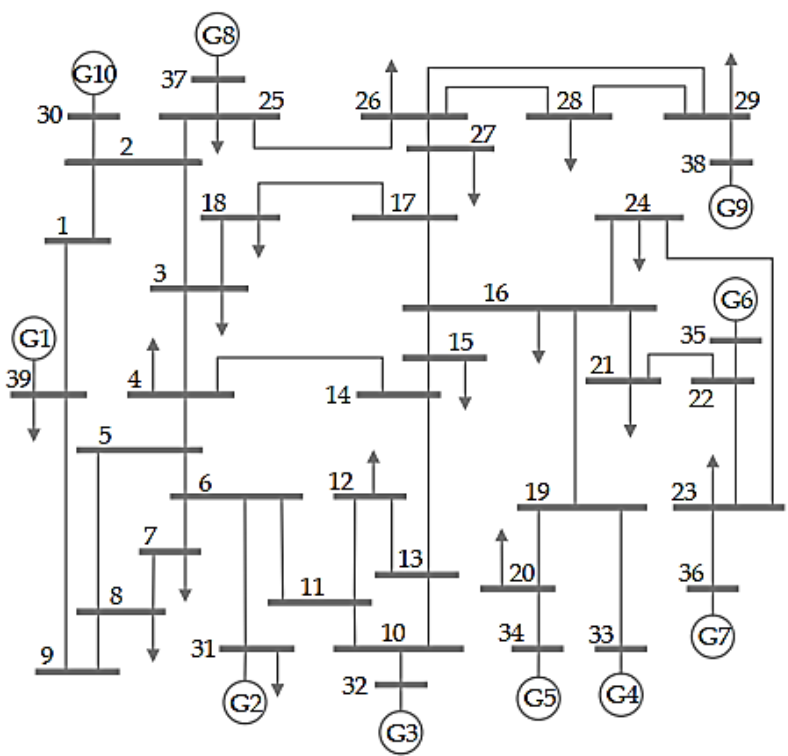

Figure 2. IEEE 39-bus test system diagram

Table 1. Data of 10 units, 39-bus IEEE system

\begin{tabular}{cccccccccccc}
\hline Unit No. & $\mathrm{P}_{\text {gimax }}$ & $\mathrm{P}_{\text {gimin }}$ & $\mathrm{a}\left(\$ / \mathbf{M W}^{2}\right)$ & $\mathrm{b}(\$ / \mathrm{MW})$ & $\mathrm{c}(\$ / \mathrm{h})$ & $\mathrm{MUTi}$ & $\mathrm{MDTi}$ & $\mathrm{HSCi}$ & $\mathrm{CSCi}$ & $\mathrm{CSHi}$ & $\mathrm{ISi}$ \\
\hline U1 & 455 & 150 & 0.00048 & 16.19 & 1000 & 8 & 8 & 4500 & 9000 & 5 & 8 \\
U2 & 455 & 150 & 0.00031 & 17.26 & 970 & 8 & 8 & 5000 & 10000 & 5 & 8 \\
U3 & 130 & 20 & 0.002 & 16.6 & 700 & 5 & 5 & 550 & 1100 & 4 & -5 \\
U4 & 130 & 20 & 0.00211 & 16.5 & 680 & 5 & 5 & 560 & 1120 & 4 & -5 \\
U5 & 162 & 25 & 0.0398 & 19.7 & 450 & 6 & 6 & 900 & 1800 & 4 & -6 \\
U6 & 80 & 20 & 0.00712 & 22.26 & 370 & 3 & 3 & 170 & 340 & 2 & -3 \\
U7 & 85 & 25 & 0.00079 & 27.74 & 480 & 3 & 3 & 260 & 520 & 2 & -3 \\
U8 & 55 & 10 & 0.00413 & 25.92 & 660 & 1 & 1 & 30 & 60 & 0 & -1 \\
U9 & 55 & 10 & 0.00222 & 27.27 & 665 & 1 & 1 & 30 & 60 & 0 & -1 \\
U10 & 55 & 10 & 0.00173 & 27.79 & 670 & 1 & 1 & 30 & 60 & 0 & -1 \\
\hline
\end{tabular}

Table 2. Load demand for 10 units system

\begin{tabular}{ccccccccccccc}
\hline Hour & 1 & 2 & 3 & 4 & 5 & 6 & 7 & 8 & 9 & 10 & 11 & 12 \\
Demand & 700 & 750 & 850 & 950 & 1000 & 1100 & 1150 & 1200 & 1300 & 1400 & 1450 & 1500 \\
Hour & 13 & 14 & 15 & 16 & 17 & 18 & 19 & 20 & 21 & 22 & 23 & 24 \\
Demand & 1400 & 1300 & 1200 & 1050 & 1000 & 1100 & 1200 & 1400 & 1300 & 1100 & 900 & 800 \\
\hline
\end{tabular}

\section{CONCLUSION}

This paper suggested a BGWOPSO algorithm to find solving to the unit commitment. The standard test system (IEEE 39-bus test systems) has been used to show the efficiency of the suggested method. From the achieved results, it is clear that the BGWOPSO algorithm is succeeded to fulfilling the minimum production cost and optimal scheduling for power generation during 24 time hours for the test systems under consideration. In terms of computation time and cost, the proposed method (BGWOPSO) outperformed the other various technologies in this analysis. The proposed algorithm reduced computing time, allowed fewer units to be started up, and had a lower scheduling cost, according to the results. This method can be used by power companies to dispatch units, and governments can use the results to promote and implement renewable energy power generation. The future work can be used the multiobjective function for minimizing the operation cost instead of a single objective function. 
Table 3. Scheduling of generation and commitment of 10 units system by BGWOPSO

\begin{tabular}{|c|c|c|c|c|c|c|c|c|c|c|c|}
\hline \multirow[b]{2}{*}{ Hour } & \multirow[b]{2}{*}{ Demand } & \multicolumn{10}{|c|}{ Generation scheduling } \\
\hline & & $\mathrm{U} 1$ & $\mathrm{U} 2$ & U3 & $\mathrm{U} 4$ & U5 & U6 & U7 & U8 & U9 & $\mathrm{U} 10$ \\
\hline 1 & 700 & 455 & 245 & 0 & 0 & 0 & 0 & 0 & 0 & 0 & 0 \\
\hline 2 & 750 & 455 & 295 & 0 & 0 & 0 & 0 & 0 & 0 & 0 & 0 \\
\hline 3 & 850 & 455 & 370 & 0 & 0 & 25 & 0 & 0 & 0 & 0 & 0 \\
\hline 4 & 950 & 455 & 455 & 0 & 0 & 40 & 0 & 0 & 0 & 0 & 0 \\
\hline 5 & 1000 & 455 & 390 & 0 & 130 & 25 & 0 & 0 & 0 & 0 & 0 \\
\hline 6 & 1100 & 455 & 360 & 130 & 130 & 25 & 0 & 0 & 0 & 0 & 0 \\
\hline 7 & 1150 & 455 & 410 & 130 & 130 & 25 & 0 & 0 & 0 & 0 & 0 \\
\hline 8 & 1200 & 455 & 455 & 130 & 130 & 30 & 0 & 0 & 0 & 0 & 0 \\
\hline 9 & 1300 & 455 & 455 & 130 & 130 & 85 & 20 & 25 & 0 & 0 & 0 \\
\hline 10 & 1400 & 455 & 455 & 130 & 130 & 162 & 33 & 25 & 10 & 0 & 0 \\
\hline 11 & 1450 & 455 & 455 & 130 & 130 & 162 & 73 & 25 & 10 & 10 & 0 \\
\hline 12 & 1500 & 455 & 455 & 130 & 130 & 162 & 80 & 25 & 43 & 10 & 10 \\
\hline 13 & 1400 & 455 & 455 & 130 & 130 & 162 & 33 & 25 & 10 & 0 & 0 \\
\hline 14 & 1300 & 455 & 455 & 130 & 130 & 85 & 20 & 25 & 0 & 0 & 0 \\
\hline 15 & 1200 & 455 & 455 & 130 & 130 & 30 & 0 & 0 & 0 & 0 & 0 \\
\hline 16 & 1050 & 455 & 310 & 130 & 130 & 25 & 0 & 0 & 0 & 0 & 0 \\
\hline 17 & 1000 & 455 & 260 & 130 & 130 & 25 & 0 & 0 & 0 & 0 & 0 \\
\hline 18 & 1100 & 455 & 360 & 130 & 130 & 25 & 0 & 0 & 0 & 0 & 0 \\
\hline 19 & 1200 & 455 & 455 & 130 & 130 & 30 & 0 & 0 & 0 & 0 & 0 \\
\hline 20 & 1400 & 455 & 455 & 130 & 130 & 162 & 33 & 25 & 10 & 0 & 0 \\
\hline 21 & 1300 & 455 & 455 & 130 & 130 & 85 & 20 & 25 & 0 & 0 & 0 \\
\hline 22 & 1100 & 455 & 455 & 0 & 0 & 145 & 20 & 25 & 0 & 0 & 0 \\
\hline 23 & 900 & 455 & 425 & 0 & 0 & 0 & 20 & 0 & 0 & 0 & 0 \\
\hline \multirow[t]{2}{*}{24} & 800 & 455 & 345 & 0 & 0 & 0 & 0 & 0 & 0 & 0 & 0 \\
\hline & & \multicolumn{10}{|c|}{ Units scheduling } \\
\hline Hour & Demand & U1 & $\mathrm{U} 2$ & U3 & $\mathrm{U} 4$ & U5 & U6 & U7 & U8 & U9 & $\mathrm{U} 10$ \\
\hline 1 & 700 & 1 & 1 & 0 & 0 & 0 & 0 & 0 & 0 & 0 & 0 \\
\hline 2 & 750 & 1 & 1 & 0 & 0 & 0 & 0 & 0 & 0 & 0 & 0 \\
\hline 3 & 850 & 1 & 1 & 0 & 0 & 1 & 0 & 0 & 0 & 0 & 0 \\
\hline 4 & 950 & 1 & 1 & 0 & 0 & 1 & 0 & 0 & 0 & 0 & 0 \\
\hline 5 & 1000 & 1 & 1 & 0 & 1 & 1 & 0 & 0 & 0 & 0 & 0 \\
\hline 6 & 1100 & 1 & 1 & 1 & 1 & 1 & 0 & 0 & 0 & 0 & 0 \\
\hline 7 & 1150 & 1 & 1 & 1 & 1 & 1 & 0 & 0 & 0 & 0 & 0 \\
\hline 8 & 1200 & 1 & 1 & 1 & 1 & 1 & 0 & 0 & 0 & 0 & 0 \\
\hline 9 & 1300 & 1 & 1 & 1 & 1 & 1 & 1 & 1 & 0 & 0 & 0 \\
\hline 10 & 1400 & 1 & 1 & 1 & 1 & 1 & 1 & 1 & 1 & 0 & 0 \\
\hline 11 & 1450 & 1 & 1 & 1 & 1 & 1 & 1 & 1 & 1 & 1 & 0 \\
\hline 12 & 1500 & 1 & 1 & 1 & 1 & 1 & 1 & 1 & 1 & 1 & 1 \\
\hline 13 & 1400 & 1 & 1 & 1 & 1 & 1 & 1 & 1 & 1 & 0 & 0 \\
\hline 14 & 1300 & 1 & 1 & 1 & 1 & 1 & 1 & 1 & 0 & 0 & 0 \\
\hline 15 & 1200 & 1 & 1 & 1 & 1 & 1 & 0 & 0 & 0 & 0 & 0 \\
\hline 16 & 1050 & 1 & 1 & 1 & 1 & 1 & 0 & 0 & 0 & 0 & 0 \\
\hline 17 & 1000 & 1 & 1 & 1 & 1 & 1 & 0 & 0 & 0 & 0 & 0 \\
\hline 18 & 1100 & 1 & 1 & 1 & 1 & 1 & 0 & 0 & 0 & 0 & 0 \\
\hline 19 & 1200 & 1 & 1 & 1 & 1 & 1 & 0 & 0 & 0 & 0 & 0 \\
\hline 20 & 1400 & 1 & 1 & 1 & 1 & 1 & 1 & 1 & 1 & 0 & 0 \\
\hline 21 & 1300 & 1 & 1 & 1 & 1 & 1 & 1 & 1 & 0 & 0 & 0 \\
\hline 22 & 1100 & 1 & 1 & 0 & 0 & 1 & 1 & 1 & 0 & 0 & 0 \\
\hline 23 & 900 & 1 & 1 & 0 & 0 & 0 & 1 & 0 & 0 & 0 & 0 \\
\hline 24 & 800 & 1 & 1 & 0 & 0 & 0 & 0 & 0 & 0 & 0 & 0 \\
\hline \multicolumn{12}{|c|}{ The total cost $=563940 \$$} \\
\hline
\end{tabular}

Table 4. Comparison total production cost between BGWOPSO and other approaches with

\begin{tabular}{clcc}
\hline No. & \multicolumn{1}{c}{ Method } & $\begin{array}{c}\text { Best generation } \\
\text { cost }(\$)\end{array}$ & $\begin{array}{c}\text { Percentage of production cost } \\
\text { for BGWOPSO to another } \\
\text { algorithms }\end{array}$ \\
\hline 1 & New Genetic Approach (NGA) [26] & 591715 & 0.046939 \\
2 & Two Stage Genetic Algorithm (TSGA) [27] & 568314.56 & 0.007694 \\
3 & GA [28] & 565866 & 0.0034036 \\
4 & PSO-LR [29] & 565835 & 0.003349 \\
5 & LR [28] & 565828 & 0.003336 \\
6 & Improved DA-PSO [1] & 565807.31 & 0.003299 \\
7 & Discrete Binary PSO [30] & 565804 & 0.003294 \\
8 & Proposed method hybrid BGWOPSO & 563940 & - \\
\hline
\end{tabular}




\section{REFERENCES}

[1] S. Khunkitti, N. R. Watson, R. Chatthaworn, S. Premrudeepreechacharn, and A. Siritaratiwat, "An improved DA-PSO optimization approach for unit commitment problem," Energies, vol. 12, 2019. doi: 10.3390/en12122335.

[2] S. Sivanagaraju and G. Sreenivasan, "Power system operation and control," Pearson, 2009.

[3] K. W. Edwin, H. D. Kochs, and R. J. Taud, "Integer programming approach to the problem of optimal unit commitment with probabilistic reserve determination," IEEE Trans. Power Appar. Syst., vol. PAS-97, no. 6, pp. 2154-2166, 1978, doi: 10.1109/TPAS.1978.354719.

[4] A. I. Cohen and M. Yoshimura, "A branch-and-bound algorithm for unit commitment," IEEE Trans. Power Appar. Syst., vol. PAS-102, no. 2, pp. 444-451, 1983, doi: 10.1109/TPAS.1983.317714.

[5] C. K. Pang, G. B. Sheble, and F. Albuyeh, "Evaluation of dynamic programming based methods and multiple area representation for thermal unit commitments," IEEE Trans. Power Appar. Syst., vol. PAS-100, no. 3, pp. 1212-1218, 1981, doi: 10.1109/TPAS.1981.316592.

[6] W. L. Snyder, H. D. Powell, and J. C. Rayburn, "Dynamic programming approach to unit commitment," IEEE Trans. Power Syst., vol. 2, no. 2, pp. 339-348, 1987, doi: 10.1109/TPWRS.1987.4335130.

[7] C. C. Su and Y. Y. Hsu, "Fuzzy dynamic programming: An application to unit commitment," IEEE Trans. Power Syst., vol. 6, no. 3, pp. 1231-1237, 1991, doi: 10.1109/59.119271.

[8] P. K. Singhal and R. N. Sharma, "Dynamic programming approach for solving power generating unit commitment problem," 2nd Int. Conf. on Comp. and Commu. Tech., 2011, pp. 298-303, doi: 10.1109/ICCCT.2011.6075161.

[9] J. A. Muckstadt and R. C. Wilson, "An application of mixed-integer programming duality to scheduling thermal generating systems," IEEE Trans. Power Appar. Syst., vol. PAS-87, no. 12, pp. 1968-1978, 1968, doi: 10.1109/TPAS.1968.292156.

[10] F. Zhuang and F. D. Galiana, "Towards a more rigorous and practical unit commitment by lagrangian relaxation," IEEE Trans. Power Syst., vol. 3, no. 2, pp. 763-773, 1988, doi: 10.1109/59.192933.

[11] A. Merlin and P. Sandrin, "A new method for unit commitment at Electricite de France," IEEE Trans. power Appar. Syst., vol. PAS-102, no. 5, pp. 1218-1225, 1983, doi: 10.1109/TPAS.1983.318063.

[12] G. B. Sheble, "Solution of the unit commitment problem by the method of unit periods," IEEE Trans. Power Syst., vol. 5, no. 1, pp. 257-260, 1990, doi: 10.1109/59.49114

[13] A. Singh and A. Khamparia, "A hybrid whale optimization-differential evolution and genetic algorithm based approach to solve unit commitment scheduling problem: WODEGA," Sustain. Comput. Informatics Syst., vol. 28, 2020, doi: 10.1016/j.suscom.2020.100442.

[14] B. Knueven, J. Ostrowski, and J. P. Watson, "On mixed-integer programming formulations for the unit commitment problem," INFORMS J. Comput., vol. 32, no. 4, pp. 857-876, Sep. 2020, doi: 10.1287/ijoc.2019.0944.

[15] Z. G. Hassan, M. Ezzat, and A. Y. Abdelaziz, "Solving unit commitment and economic load dispatch problems using modern optimization algorithms," Int. J. Eng. Sci. Technol., vol. 9, no. 4, 2017, doi: 10.4314/ijest.v9i4.2.

[16] M. Farsadi, H. Hosseinnejad, and T. S. Dizaji, "Solving unit commitment and economic dispatch simultaneously considering generator constraints by using nested PSO," ELECO 2015 - 9th Int. Conf. Electr. Electron. Eng., 2016, pp. 493-499, doi: 10.1109/ELECO.2015.7394478

[17] M. Tuffaha and J. T. Gravdahl, "Dynamic formulation of the unit commitment and economic dispatch problems," Proc. IEEE Int. Conf. Ind. Technol., 2015, vol. 2015, pp. 1294-1298, doi: 10.1109/ICIT.2015.7125276.

[18] Y. Zhai, X. Liao, N. Mu, and J. Le, "A two-layer algorithm based on PSO for solving unit commitment problem," Soft Comput., vol. 24, no. 12, pp. 9161-9178, 2020, doi: 10.1007/s00500-019-04445-X.

[19] A. J. Wood, B. F. Wollenberg, and G. B. Sheble, "Power generation, operation, and control," 3rd ed, Wiley, New York, 2014.

[20] J. Kennedy and R. C. Eberhart, "Particle swarm optimization," Proceedings of the IEEE International Conference on Neural Networks, Perth, Australia, vol. 4, 1995, pp. 1942-1948.

[21] P. Sriyanyong and Y. H. Song, "Unit commitment using particle swarm optimization combined with lagrange relaxation," IEEE Power Eng. Soc. Gen. Meet., vol. 3, no. 6, pp. 2752-2759, 2005, doi: 10.1109/pes.2005.1489390.

[22] S. Mirjalili, S. M. Mirjalili, and A. Lewis, "Grey wolf optimizer," Adv. Eng. Softw., vol. 69, pp. 46-61, 2014, doi: 10.1016/j.advengsoft..12.007.

[23] V. K. Kamboj, "A novel hybrid PSO-GWO approach for unit commitment problem," Neural Comput. Appl., vol. 27, no. 6, pp. 1643-1655, 2016, doi: 10.1007/s00521-015-1962-4.

[24] Q. Al-Tashi, S. J. A. Kadir, H. M. Rais, S. Mirjalili, and H. Alhussian, "Binary optimization using hybrid grey wolf optimization for feature selection," IEEE Access, vol. 7, pp. 39496-39508, 2019, doi: 10.1109/ACCESS.2019.2906757.

[25] N. Singh and S. B. Singh, "Hybrid algorithm of particle swarm optimization and grey wolf optimizer for improving convergence performance," J. Appl. Math., vol. 2017, 2017, doi: 10.1155/2017/2030489.

[26] D. Ganguly, V. Sarkar, and J. Pal, "A new genetic approach for solving the unit commitment problem," 2004 Int. Conf. Power Syst. Technol. POWERCON 2004, vol. 1, pp. 542-547, 2004, doi: 10.1109/icpst.2004.1460054.

[27] A. S. Eldin, M. A. H. El-sayed, and H. K. M. Youssef, "A two-stage genetic based technique for the unit commitment optimization problem," 12th Int. Middle East Power Syst. Conf. 2008, pp. 425-430D.

[28] D. N. Simopoulos, S. D. Kavatza and C. D. Vournas, "Unit Commitment by an Enhanced Simulated Annealing Algorithm," 2006 IEEE PES Power Systems Conference and Exposition, 2006, pp. 193-201, doi: 10.1109/PSCE.2006.296296.

[29] H. H. Balci and J. F. Valenzuela, "Scheduling electric power generators using particle swarm optimization combined with the Lagrangian relaxation method," Int. J. Appl. Math. Comput. Sci., vol. 14, no. 3, pp. 411-421, 2004

[30] Z. L. Gaing, "Discrete particle swarm optimization algorithm for unit commitment," 2003 IEEE Power Eng. Soc. Gen. Meet. Conf. Proc., vol. 1, pp. 418-424, 2003, doi: 10.1109/pes.2003.1267212. 